\title{
Current concepts in biomaterials in dental implant
}

\author{
Suraksha Shrestha*, Sarita Joshi \\ Department of Prosthodontics, National Academy of Medical Sciences, Bir Hospital, Kathmandu, Nepal \\ Email address: \\ suraksha_shrestha@hotmail.com (S. Shrestha), saritajoshipradhan@yahoo.com (S. Joshi)
}

\section{To cite this article:}

Suraksha Shrestha, Sarita Joshi. Current Concepts in Biomaterials in Dental Implant. Science Research. Vol. 2, No. 1, 2014 , pp. 7-12. doi: 10.11648/j.sr.20140201.12

\begin{abstract}
Myriads of materials have been used for replacement of missing teeth through implantation. The success of these materials depend on the ability to integrate with the host environment showing biological compatibility, mechanical compatibility, and morphological compatibility to the surrounding vital tissues. Certain materials have shown this promising property and have been used in dental implantology. With recent advances in technology, these materials are better able to improve fixation to bone through various surface modifications and bioengineering.
\end{abstract}

Keywords: Biocompatibility, Dental Implant, Surface Modification

\section{Introduction}

Replacement of missing tooth with various materials dates back to ancient period of Greek and Egyptian civilization where bone, carved ivory, shells, metal and even animal teeth were used. Many materials were introduced later on but unpredictable failures occurred with them due to the lack of firm attachment. In 1952, Dr Perr Ingvar Branemark developed a threaded implant design made of pure titanium that showed direct contact with bone. This phenomenon was called osseointegration, defined by the American Academy of Implant Dentistry as "the firm, direct and lasting biological attachment of a metallic implant to vital bone with no intervening connective tissue" [1]. With the emerging concept of osseointegration, devices were designed to mimic as much as possible cell interactions that normally take place during bone remodeling. Currently the implant materials available are diverse. Different materials, such as platinum, silver, steel, cobalt alloys, titanium and alloys, acrylic, carbon, sapphire, alumina, tantalum, niobium, zirconia and calcium phosphate compounds have been used as dental implant material [2].

\section{Titanium as an Implant Material}

The evolution of titanium as biomaterial for implant has dramatically increased in past few years because of its favorable combination of mechanical strength, chemical stability, and biocompatibility [3].

Commercially pure titanium (CpTi) and extra low interstitial Ti-6Al $4 \mathrm{~V}$ (ELI) are the two most common titanium based materials. The first generation with a history of 50 years of success consisted of titanium implants, which were machined with a smooth surface texture. As the implant surfaces were recognized to play an important role in molecular interactions, cellular response and osseointegration, second generation implants with surfaces which can accelerate and improve implant osseointegration were developed. These second generation of clinically used implants underwent mechanical blasting coupled or not, with acid etch, bioactive coatings, anodized and, more recently, laser modified surfaces [4].

Pure titanium is a rather soft nonmagnetic material. It undergoes a crystallographic change from alpha to beta phase on heating to $883^{\circ} \mathrm{C}$. Phase stabilizers like aluminium and vanadium are added to improve the mechanical properties of this metal. Aluminum called as alpha-phase condition stabilizer serves to increase the strength and decrease the weight of the alloy. Vanadium called as beta-phase stabilizer helps increase the ductility. The most common alloy contains $6 \%$ aluminum and $4 \%$ vanadium called as Ti-6Al-4V. They are light, strong, and highly resistant to fatigue and corrosion. Although they are stiffer than bone, their modulus of elasticity (stiffness) is closer to bone than any other important implant metal. This property leads to a more even distribution of stress at the critical bone-implant interface because the bone and implant will flex in a more similar fashion.

Recently due to the local adverse tissue reaction and immunological responses niobium $(\mathrm{Nb})$ has replaced vanadium, and $\mathrm{Ti}-6 \mathrm{Al} 7 \mathrm{Nb}$ has been proposed as an 
alternative. Other elements like zirconium, tantalum, palladium and indium are also being explored for their ability to match the mechanical strength and corrosion resistance of Ti-6Al 4V, with improved biocompatibility [5].

\section{Corrosion in Implant}

Titanium forms a tenacious oxides layer in air or oxygenated solution of about $10-100 \mathrm{~A}^{0}$ within a minute and will repair itself instantaneously on damage as might occur during insertion of an implant. This layer helps in passivation and makes it corrosion resistant. However, accumulation of titanium in tissue can be observed. The normal level of Ti in human tissue is $50 \mathrm{ppm}$. Values of 100 to $300 \mathrm{ppm}$ are frequently observed in soft tissues surrounding $\mathrm{Ti}$ implants. At these levels, tissue discoloration with $\mathrm{Ti}$ pigments can be seen. This rate of dissolution is one of the lowest of all passivated implant metals and seems to be well tolerated by the body. In a study done, a newly developed beta titanium alloy i.e Ti $24 \mathrm{Nb} 4 \mathrm{Zr} 7.9 \mathrm{Sn}$ (TNZS) is considered a suitable dental implant due to its low modulus of elasticity and high strength. The corrosion behaviour of TNZS alloy was studied through static immersion in various simulated physiologic test solutions and compared with CpTi and $\mathrm{Ti}$ $6 \mathrm{Al} 4 \mathrm{~V}$. Results showed that quantity of each metal element released from TNZS alloy into fluoridated solution was much higher than non fluoridated solution. The total elemental release from TNZS was lower than CpTi and Ti $6 \mathrm{Al} 4 \mathrm{~V}$ in the same solution [6].

\section{Surface Treatment of Titanium Implant}

Though implant osseointegration takes place several months, the bone implant contact averages $70-80 \%$ with minimum of $60 \%$ even for successful implant that lasted for 17 years which lead to more areas of manipulation for improvement on surfaces for better osseointegration [7].

Previously implants had macro-irregularities like macroscopic threads, fenestrations, pores, grooves, steps, threads, or other surface irregularities that were visible. The idea was to create mechanical interlocking between implant and bone at the macro level. However, difficulty in achieving initial stability, post implantation relative motion, adverse interfacial bone remodeling all lead to search for improvement of the surface quality of a titanium dental implant in terms of the rate and strength of its osseointegration.

At the microscopic level, surface irregularities are at that level, possibly in conjunction with macro-irregularities. This would afford the possibility of microscopic interlocking of bone and implant, which might enhance the load transmitting capabilities of the interface. Microscopic level involves surface coatings and modification of surface coatings and modification of surface topography to enhance bone implant integration. Nowadays, the improvement of the bone forming activity at the interface is committed to nanoscale features that have the ability to induce the differentiation of stem cells along the osteogenic pathway and help cell attraction and adhesion to extracelluar matrix causing clustering of integrins into focal adhesion complexes (FA), and activate intracellular signaling cascade all leading to a better osseointegration [8].

Various techniques have been used to create nanofeatures on dental implant. Nanomaterials are essentially polymers reinforced by nanoparticles resulting in novel materials which can be used as light weight replacements for metals. When brought into a bulk material, nanoparticle can strongly influence the mechanical properties of the materials. Chemical methods include anodic oxidation in which nanostructure with diameter of $<100 \mathrm{~nm}$ is created on Ti implants through electrolytic reaction that takes place at the $\mathrm{Ti}$ anode, resulting in the growth of an oxide film. This results in a surface with micropores which demonstrate increased cell attachment [9]. Blasting implant surfaces with particles of various diameters is one of the frequently used methods of surface alteration in which aluminium oxide, titanium oxide and calcium phosphate with particle size ranging from small, medium to large (150- $350 \mu \mathrm{m})$ grit are used. Clinical studies have shown higher marginal bone levels and survival rates for blasted implant than machine turned implants [10]. Studies have presented mixed result regarding aluminium oxide left after blasting. Few authors have reported catalization of osseointegration while others have shown impaired bone formation by a possible competitive action with calcium ions [11].

Etching with strong acid produces micropits $(0.5-2 \mu \mathrm{m})$ in diameter. Dual acid etching with $\mathrm{HCl}$ and $\mathrm{H}_{2} \mathrm{SO}_{4}$ heated above $100^{\circ} \mathrm{C}$ has produced surface topography able to attach to fibrin scaffold and promote adhesion of osteogenic cells. Sandblasting and acid etching (SLAsandblasted, large grit, acid etched surface) is produced by large grit $(250-500 \mu \mathrm{m})$ blasting followed by etching with acids which also produces rough surface, microtexturing and cleaning and better bone integration [12]. Plasma spraying gives a porous surface that bone can penetrate more readily and enhance osseointegration. Titanium plasma spraying consists of injecting titanium powder into a plasma torch at high temperature where particles are projected on to the surface of implants where they condense and fuse together forming a film about $100 \mathrm{~nm}$ [13]. It has been shown that this 3 dimensional topography increased tensile strength at the bone implant interface and have often been recommended for regions with low bone density.

Fluoride (F) treatment- Titanium is very reactive to fluoride forming soluble titanium fluoride in $\mathrm{F}$ solution. This treatment enhances osseointegration and osteoblastic differentitation with increased expression of Cbfal, osterix and bone sialoprotein [14]. Fluoridated rough implants also 
withstood greater push-out forces and showed a significantly higher removal torque than control implants. However, detrimental effect of $F$ on the corrosion resistance of titanium and titanium alloys has been extensively reported. Fluorides are very aggressive on the protective oxide formed on titanium and titanium alloys [15].

\section{Biomodification of Titanium Implant}

The addition of calcium and phosphate based materials as coatings have received significant attention as these are components of natural bone. Plasma sprayed hydroxyapatite (PSHA) coating on titanium implant lead to improved maturation of newly formed bone tissue due to the high biocompatibility and osteoconduction of calcium phosphate materials and has been widely used for different hard tissue application such as hydroxyapatite (HA) coated metallic implants and bone substitute materials. HA coating is mainly indicated in type 4 bone (Misch and Judy), fresh extraction socket and newly grafted sites. HA coatings have higher success rates in maxilla where it helps to achieve primary stability as it lowers corrosion rates and enables to obtain improved bone implant attachment. However, sometimes delamination or dissolution of coating may lead to implant failure [16].

Recently, laser deposition/ablation process results in titanium surface microstructure with greatly increased hardness, corrosion resistance, and a high degree of purity with a standard roughness and thicker oxide layer. Biological studies evaluating the role of titanium ablation topography and chemical properties showed the potential of the grooved surfaces to orient osteoblast cell attachment and control the direction of in growth [14]. Using histomorphometrical analysis, the effects of titanium surface modification by laser ablation (Nd: YAG) followed by thin chemical deposition of HA was studied. The result showed that HA biomimetic coating preceded by laser treatment induced the contact osteogenesis and allowed the formation of a more stable bone-implant interface even in earlier periods [17].

\section{Sputter deposition}

Sputtering is a process whereby atoms or molecules of a material are ejected in a vacuum chamber by bombardment of high energy ions. Radiofrequency magnetron sputtering is a magnetically enhanced variant of diode sputtering used to deposit thin films of calcium phosphate coatings on titanium implants. Studies have shown that these coatings were more retentive, with the chemical structure being precisely controlled [18]. Magnetron sputtering is a viable thin film technique as it allows the mechanical properties of titanium to be preserved while maintaining bioactivity of the coated HA. An outward diffusion of titanium into HA layer, forming $\mathrm{TiO}_{2}$ at the interface shows strong bonding between coating and titanium.
Various attempts have been made to improve and accelerate osseointegration by bioactive factor incorporation to titanium surfaces. Of these, osteogenic drugs, antiresoprtive drugs, such as biphosphonates are very useful in clinical cases with deficient bone support. Increased bone density locally in peri-implant region has been demonstrated in PSHA implant immersed in pamidronate or zoledronate.

\section{Antibiotic Coatings}

Antibacterial coatings on the surface have been studied as a possible way to prevent surgical site infections. Gentamycin along with the layer of HA can be coated onto the implant surface which may act as a local prophylactic agent along with the systemic antibiotics in dental implant surgery. Study was done to investigate if different $\mathrm{pH}$, atmosphere and surface properties could restrict bacterial adhesion to titanium surfaces used in dental implants. Titanium discs with machined or anodized (TiUnite ${ }^{\mathrm{TM}}$ ) surface were incubated with a co-culture of Streptococcus mitis and Actinomyces oris (early colonizers of oral surfaces) at $\mathrm{pH}$ 5.0, 7.0 and 9.0 at aerobic or anaerobic atmosphere. The adhesion was analysed by counting colony forming units (CFU) on agar and by confocal laser scanning microscopy (CLSM). The results found that bacterial adhesion by $\mathrm{S}$. mitis and $\mathrm{A}$. oris can be restricted by acidic $\mathrm{pH}$ and aerobic atmosphere. The anodized surface reduced the adhesion of $\mathrm{S}$. mitis compared to the machined surface; while A. oris adhered equally well to the pores of the anodized surface and to the grooves of the machined surface [19].

\section{Decontamination of Implant Surfaces}

Tetracycline- $\mathrm{HCl}$ treatment has been regarded as a practical and effective chemical modality for decontamination and detoxification of contaminated implant surfaces. It also effectively removes the smear layer as well as endotoxins from the implant surface. Further, it inhibits collagenase activity, increases cell proliferation as well as attachment and bone healing. Tetracycline also enhances blood clot attachment and retention on the implant surface during the initial phase of the healing process and thus promotes osseointegration [20].

\section{Ceramic Biomaterials}

\section{i) Bioinert ceramics}

Oxide ceramics were introduced for surgical implant devices because of their inertness to biodegradation, high strength, physical characteristics such as color and minimal thermal and electrical conductivity [21]. Ceramics have been used in bulk forms, and more recently as coatings on metals. Earlier, aluminium oxide used was shown to possess high biocompatibility and microscopically highly mineralized mature compact lamellar bone with no 
connective tissue or inflammatory cells present at the interface. Despite its good osseointegration, it was withdrawn from the market because of its poor survival rate [22].

With recent advances in implantable biomaterials research and technology, bioceramics such as zirconia (zirconium dioxide) are now available. Zirconium undergoes an oxidation and crystallization process which allows it to transition into a structurally stable and inert crystal. This bioceramic crystal is called Yttrium Stabilized Tetragonal Zirconium Polycrystal (Y-TZP) also called zirconium dioxide. The strength and toughness of zirconia can be accounted for by its toughening mechanisms, such as crack deflection, zone shielding, contact shielding, and crack bridging. Prevention of crack propagation is of critical importance in high-fatigue situations, such as those encountered in mastication and parafunction [23]. This combination of favorable mechanical properties makes zirconia a unique and stable material for use in high-load situations. Zirconia is radioopaque and clearly visible on radiographs. Its ivory color is similar to color of natural teeth and is especially critical in the esthetic zone with high lip line smiles. Furthermore, with the development of dental CAD CAM systems, this high strength ceramic is becoming the first choice in treating esthetic implant cases. Besides these favorable properties, zirconia is proposed to accumulate lesser plaque than titanium [24].

A number of studies have been done to compare osseointegration of zirconia with that of titanium implants. Most studies have revealed no significant differences between the two and found similar attachment of both implants to bone, with similar features ultrastructurally [25]. However, some studies also reported higher bone to implant contact (BIC) with zirconia than titanium and an increased proliferation of osteoblasts was found around zirconia compared to titanium [26]. Periodontal aspect shows less bleeding on probing and less amount of recession with zirconia than with titanium implant [27].

Because of the lack of clinical reports on the long term success rates with zirconia implants, caution with regard to certain aspects of zirconia implants, such as tensile strength and modulus of elasticity should be considered. Nevertheless, studies in relation to its clinical use in view of its good osseointegration, esthetics and biocompatibility are supportive.

ii) Bioactive ceramic

Besides uses such as bone substitute and drug delivery vehicle, calcium phosphates have also been considered good option for implant coatings that may promote accelerated bone healing around implants [28]. Calcium phosphate materials (CaPs), such as TCP (tricalcium phosphate), glass ceramics, hydrooxyapatite are included in this category. These have excellent biocompatibility, no local or systemic toxicity, minimal thermal and electrical conductivity, no alteration to natural mineralization process of bone, lower mechanical tensile, shear and fatigue strength. Based on numerous in vitro and vivo experiments, it was apparent that dense or porous HA ceramics could be considered to be long term or permanent bone implant materials, whereas porous TCP ceramic could potentially serve as bioresorable.

With a CaPs coating, metallic implants can be alternatively regarded as scaffolds for bone-forming cells that can further enhance early and strong fixation of a bonesubstituting implant by stimulating bone formation starting from the implant surface [29]. Most successful method to apply CaPs coatings to implants to date seems to be the plasma-spraying technique, due to its high deposition rate and ability to coat large areas. It is the only coating method that has been used for titanium dental implants in clinical practice.

On the basis of study done for evaluation of $\mathrm{CaP}$ coatings on osteoconduction, results showed that the addition of a thin layer of $\mathrm{CaP}$ to the implant promotes accelerated bone healing around porous-surfaced implants-even after only 2 weeks of initial healing [30]. Osteoporotic conditions have also shown good result with integration if coated with calcium phosphates [31]. Implant today can be coated with biomimetic technology, and thus bioactive agents, growth factors, BMPs, osteogenic drugs can be incorporated into $\mathrm{CaP}$ into the three-dimensional crystal latticework from which they will be gradually released in vivo as the layer undergoes degradation [32].

Due to their self-hardening and appropriate mechanical properties, high osteoconductivity, excellent surface chemistry and surface topography to bone defect surfaces, CaP-based biomaterials can be used with outstanding results in a number of dental applications, including ridge augmentation, implant coating, bone defect fill and sinus lift [33].

\section{Carbon and Carbon Silicone Compounds}

Carbon based biomaterials which elicits minimal host respone have also been used for ceramic like coatings on metallic implants. In vitro study has shown better cell attachment on carbon coated zirconia than uncoated disc [34]. Unlike metals, polymers and other ceramics, these carbonaceous materials do not suffer from fatigue. Their intrinsic brittleness and low tensile strength limits their use in major load bearing applications. However in one type of carbon blade type of dental implant, fracture loads were shown to be higher than forces expected in mastication [35].

\section{Polymers and Composites}

Polymeric implants in the form of polymethylmethacrylate and polytetrafluoroethylene were first used in 1930s. However, low mechanical strength of polymers has precluded their use as implant materials. Combination of polymers and other categories of synthetic biomaterials ( $\mathrm{HA}, \mathrm{Al}_{2} \mathrm{O}_{3}$, Glass ceramics) have been used 
in porous or solid forms for tissue attachment, replacement and augumentation as coatings to transfer force to soft and hard tissue region. Biodegradable polymers such as Polyvinyl alcohol, polylactides or glycosides, cyanoacrylates or other hydrated forms have been combined with biodegradable CaPO4 for use such as structured scaffolds, plates, screws or other such applications such as bone augmentation and periimplant bone defect repairs. The use of polymers for osseointegrated implant is confined to components between prosthesis and implant for shock absorption and better simulates the biomechanical function of natural tooth function. The IMZ implants are either titanium plasma sprayed or hydroxyapatite coated and incorporate a polymethylene intramobile element (IME) which acts as an internal shock absorber and is placed between the implant and prosthesis through IMC (intamoblie connector) to initiate mobility and ensure a more uniform stress distribution along bone implant interface. Studies have demonstrated that this shock absorbing element also helps in reducing occlusal loads.

\section{Future Trends}

The arrival of nanotechnology has opened new opportunities for manipulation of implant surfaces. In recent years, development of nanostructured ceramic materials like polymer nanocomposites (PNC) offers an attractive path to the development of new implant materials directly from a computer model with determined shapes and porosities. However, at a more basic level, it is still not completely clear that nanopatterning will be substantially better than patterning at micron scale. High density of nanopillars has shown to create a superhydrophobic surface that can be detrimental and most of the basic studies have only been performed on flat surface.

The optimum coating composition and its thickness depending on specific location, the biodegradation rates of different coating materials based on load bearing, influence of surface topography and chemistry of dental implants controlling cell response etc. will provide the information needed to design and engineer a library of dental implants for treating diverse population of patients.

\section{Conclusion}

The primary objective of any implant system is to have firm fixation to the bone and this could be influenced by biomechanical as well as biomaterial selection. Titanium has long been regarded as a biocompatible implant material and recently various modification of its surface has been emerging at molecular and atomic level to enhance osseointegratition. Bioceramics, besides being esthetic, its biomemetic phenomenon has also been incorporated for better bone implant contact.

\section{References}

[1] Branemark PI, Hansson BO, Adell R, et al . Osseointegrated implants in the treatment of the edentulous jaw. Experience from a 10 year period. Scand J Plast Reconstr Surg Suppl $1977 ; 16: 1-132$.

[2] Albrektsson T, Wennerberg A. The impact of oral implants past and future, 1966-2042. J Can Dent Assoc 2005;71(5):327.

[3] Brunette DM, Tengvall P, Textor, M \& Thomsen P. Titanium in medicine: material science, surface science, engineering, biological responses, and medical applications. Berlin, Germany: Springer (eds) 2001.

[4] Brånemark R, Emanuelsson L, Palmquist A \& Thomsen P. Bone response to laser induced micro- and nano-size titanium surface features. Nanomedicine 2011;7(2):220-7.

[5] Naganawa T, Ishihara Y, Iwata T, Koide A, Ohguchi M, Ohguchi Y. In vitro biocompatibility of a new titanium29niobium-13tantalum-4.6zirconium alloy with osteoblast-like MG63 cells. J Periodontol 2004;75:17011707.

[6] Cheng Y, Hu J, Zhang C, Wang Z, Hao Y, Gao B. Corrosion behavior of novel Ti-24Nb-4Zr-7.9Sn alloy for dental implant applications in vitro. J Biomed Mater Res B Appl Biometer 2013 Feb;101(2):287-94.

[7] T Albrektsson, A R Eriksson, B Friberg et al., "Histologic investigations on 33 retrieved nobelpharma implants," Clinical Materials 1993;12(1):1-9.

[8] Monsees TK, Barth K, Tippelt S, et al. Surface patterning on adhesion, differentiation, and orientation of osteoblast-like cells. Cell Tiss Org 2005;180:81-95.

[9] Gupta, A.; Dhanraj, M. \& Sivagami, G. Status of surface treatment in endosseous implant: a literary overview. Indian journal of dental research 2010;21:433-438.

[10] Gotfredsen K, Karlsson U. A prospective 5-year study of fixed partial prostheses supported by implants with machined and TiO2-blasted surface. J Prosthodont 2001;10:2-7.

[11] Cochran DL, Nummikoski PV, et al. Evaluation of an endosseous titanium implant with sandblasted and acidetched surface in the canine mandible: Radiographic results. Clin Oral Implants Res 1996;7:240-52.

[12] Orsini G, Assenza B, Scarano A, Piattelli M, Piattelli A.Surface analysis of machined versus sandblasted and acid etched titanium implants. Int J Oral Maxillofac Implants 2000;15:779-84.

[13] Reising A, Yao C, Storey D, Webster T J. Greater osteoblast long-term functions on ionic plasma deposited nanostructured orthopedic implant coatings. J Biomed Mater Res A 2008;87:78-83.

[14] Cooper LF, Zhou Y, Takebe J, Guo J, Abron A, Holmen A \& Ellingsen JE. Fluoride modification effects on osteoblast behavior and bone formation at $\mathrm{TiO} 2$ gritblasted c.p. titanium endosseous implants. Biomaterials 2006;27:926-936. 
[15] Titanium applications in dentistry, ADA council on scientific affairs. JADA 2003;134:347-49.

[16] Misch Contemporary Implant Dentistry. $3^{\text {rd }}$ ed Mosby .2008 . p 614.

[17] Faeda RS, Spin-Neto R, Marcantonio E, Guastaldi AC, Marcantonio E Jr. Laser ablation in titanium implants followed by biomimetic hydroxyapatite coating: Histomorphometric study in rabbits. Microsc Res Tech. 2012;75(7):940-8.

[18] McCafferty MM, Burke GA, Meenan BJ. Mesenchymal stem cell response to conformal sputter deposited calcium phosphate thin films on nanostructured titanium surfaces. $\mathbf{J}$ Biomed Mater Res A 2013 Nov 1. doi: 10.1002/jbm.a.35018. [Epub ahead of print]

[19] Caous JS, Lövenklev M, Fäldt J, Langton M. Adhesion of Streptococcus mitis and Actinomyces oris in co-culture to machined and anodized titanium surfaces as affected by atmosphere and pH. BMC Oral Health 2013 Jan 8;13:4. doi: $10.1186 / 1472-6831-13-4$.

[20] Herr Y, Woo J, Kwon Y, Park J, Heo S \& Chung J. Implant Surface Conditioning with Tetracycline-HCl: A SEM Study. Key Engineering Materials 2008;361:849-852.

[21] Vincenzini P, editor: Ceramics in surgery, Amsterdam, 1983, Elsevier.

[22] Berge TI, Gronningsaeter AG. Survival of single crystal sapphire implants supporting mandibular overdentures. Clin Oral Implants Res 2000;11(2):154-62.

[23] Dunn DB. The use of a zirconia custom implant supported FPD prosthesis to treat implant failure in anterior maxilla: A clinical report J Prosthet Dent 2008;100:415-21.

[24] Van Brakel R, Cune MS, van Winkelhoff AJ, de Putter C, Verhoeven JW, van der Reijden W. Early bacterial colonization and soft tissue health around zirconia and titanium abutments: An In vivo study in man. Clin Oral Impl Res 2011;22:571-7.

[25] Gahlert M, Rohling S, Wieland M, Sprecher CM, Kniha H, Milz S. Osseointegration of zirconia and titanium dental implants: A histological \& histomorphometrical study in the maxilla of pigs. Clin Oral Implants Res 2009;20:1247-53.

[26] Dubruille JH, Viguier E, Le Naour G, Dubruille MT, Auriol M, Le Charpentier Y. Evaluation of combinations of titanium, zirconia, and alumina implants with 2 bone fillers in the dog. Int J Oral Maxillofac Implants 1999;14:271-7.

[27] Tete S, Mastrengelo F, Bianchi A, Zizzari V, Scarano A. Collagen fiber orientation around machined titanium and zirconia dental implant necks: An animal study. Int J Oral Maxillofac Implants 2009;24:52-8.

[28] Daculsi G. Biphasic calcium phosphate concept applied to artificial bone, implant coating and injectable bone substitute. Biomaterials.1998;19:1473-8.

[29] de Jonge LT, Leeuwenburgh SC, Wolke JG, Jansen JA. Organicinorganic surface modifications for titanium implant surfaces. Pharm Res 2008;25:2357-69.

[30] The effect of calcium phosphate implants coating on osteoconduction. Yang C. Oral Surg Oral Med Oral Pathol Oral Radiol Endod 2001 Dec;92(6):606-9.

[31] Alghamdi HS, Cuijpers VM, Wolke JG, van den Beucken JJ, Jansen JA. Calcium-phosphate-coated oral implants promote osseointegration in osteoporosis. J Dent Res 2013;92(11):982-8

[32] Liu Y, Huse RO, de Groot K, Buser D, Hunziker EB. Delivery mode and efficacy of BMP-2 in association with implants. J Dent Res. 2007;86:84-9.

[33] Paital SR, Dahotre NB. Calcium phosphate coatings for bio implant applications: materials, performance factors, and methodologies. Mater Sci Eng R 2009;66:1-70.

[34] Kou W, Akasaka T, Watari F, Sjögren G. An in vitro evaluation of the biological effects of carbon nanotubecoated dental zirconia. ISRN Dent 2013 August 20;2013:296727. doi: 10.1155/2013/296727.

[35] Shim HS. The mechanical behavior of LTI carbon dental implants. Biomater Med Devices Artif Organs 1976;4(2):181-92. 